\title{
Estimation methods for human circadian phase by use of peripheral tissues
}

\author{
Ritsuko Matsumura ${ }^{1}$, Koichi Node ${ }^{2}$ and Makoto Akashi ${ }^{1}$ \\ Almost all living organisms, including humans, exhibit diurnal rhythms of physiology and behavior, which are driven by the \\ circadian clock. Many studies have found that chronic misalignment between circadian and environmental/social rhythms carries \\ a significant risk of various disorders, including sleep disorders, metabolic syndrome, cardiovascular diseases and cancer. \\ However, irregular sleep-wake cycles and circadian maladjustment often cause 'social jet lag', which is minor but chronic jet-lag \\ in our daily lives. Establishment of objective and convenient circadian-phase estimation methods in the clinical setting would \\ therefore greatly contribute not only to resolving this global health problem but also to developing chronomedicine, a clinical \\ approach for optimizing the time of day of treatments. Traditional melatonin-based methods have limitations with respect to \\ circadian-phase evaluation; however, estimation methods based on clock gene expression may be able to compensate for these \\ limitations. As a representative application of circadian-phase estimation based on clock gene expression, our method of using \\ hair follicle cells may aid in the rapid clinical detection of circadian-related sleep problems, especially circadian rhythm sleep \\ disorders that are masked because of forced adaptation to social time schedules.
} Hypertension Research (2016) 39, 623-627; doi:10.1038/hr.2016.68; published online 23 June 2016

Keywords: circadian rhythm; clock gene; social jet lag; sleep disorder

\section{WHY OBJECTIVE ESTIMATION OF THE HUMAN CIRCADIAN PHASE IS IMPORTANT}

Almost all living organisms exhibit diurnal rhythms in physiology and behavior, which are driven by an internal oscillator, the circadian clock. ${ }^{1,2}$ The molecular clockwork consists of the clock gene-driven and autonomous negative feedback loops of transcription. ${ }^{3,4}$ These transcriptional feedback loops generate the circadian expression of a wide range of genes, which in turn produces circadian rhythms in diverse biological processes. ${ }^{5-8}$ The circadian clock enables gene expression at appropriate times of the day, allowing organisms to adapt to the earth's rotation.

It has been reported that chronic misalignment between circadian and environmental/social rhythms carries a significant risk of various disorders, including sleep disorders, metabolic syndrome, cardiovascular diseases and cancer. ${ }^{9-15}$ In humans, the main contributors to circadian misalignment are irregular sleep-wake cycles and circadian maladjustments induced by chronic exposure to artificial light environments (poor sunlight and excessive night light). ${ }^{16-18}$ The best example is rotating shift work. ${ }^{19-21}$ Therefore, the objective estimation of human circadian phases may be useful in the prevention of circadian rhythm-related diseases.

Chronomedical treatment in clinical settings also requires convenient methods for monitoring human circadian phases (Figure 1); to ensure effective chronomedicine, medication should be taken during periods when the biological activity of the pharmacological targets is high and/or when the activity of the nonspecific targets responsible for side effects is low. ${ }^{22,23}$ Because modern life environments and lifestyle habits have enhanced the differences in the circadian phases among individuals, estimation of individuals' internal phases is required to optimize the timing of medication administration.

\section{BENEFITS AND LIMITATIONS OF MELATONIN-BASED CIRCADIAN-PHASE ESTIMATION}

Melatonin is believed to be a far more reliable circadian marker than other traditional markers deduced from sleep diaries, actograms and core temperatures. ${ }^{24-27}$ Dim light melatonin onset (DLMO) has widely been used to evaluate the human circadian phase, and substantial data have been accumulated by a large number of studies using DLMO. ${ }^{24,25,28,29}$ However, this method has several limitations, as described below (Figure 2). First, endogenous melatonin, a small indoleamine, is a pineal hormone whose role in the autonomous circadian oscillator remains unclear; ${ }^{30,31}$ therefore, endogenous melatonin may not be a direct indicator of the phase of the molecular oscillator. Second, research subjects must spend at least several hours under dim light conditions because melatonin concentration dramatically decreases in response to light input via the eyes, ${ }^{32}$ and the collection duration of saliva or blood must be extended to more than $5 \mathrm{~h}$ when a rough prediction of DLMO is difficult because of subjects' irregular sleep-wake cycles. This light-controlled

\footnotetext{
${ }^{1}$ The Research Institute for Time Studies, Yamaguchi University, Yamaguchi, Japan and ${ }^{2}$ Department of Cardiovascular Medicine, Saga University, Saga, Japan Correspondence: Professor M Akashi, The Research Institute for Time Studies, Yamaguchi University, 1677-1 Yoshida, Yamaguchi 753-8511, Japan.

E-mail: akashima@yamaguchi-u.ac.jp

Received 29 March 2016; revised 14 April 2016; accepted 15 April 2016; published online 23 June 2016
} 


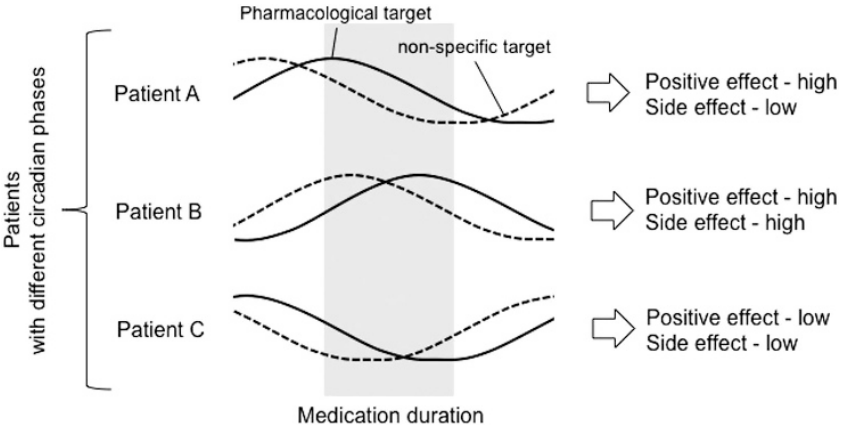

Figure 1 Requirement of circadian-phase estimation to optimize the timing of medication. Chronomedicine works when the biological activity of pharmacological targets or of nonspecific targets responsible for side effects has diurnal rhythms. Because modern life environments and lifestyle habits have enhanced the individual differences in the circadian phases, the activity of both targets oscillates in a different phase among individuals. If a medication is taken at the same time of the day without regard for the differences of the circadian phases of the individual patients, the positive effects and the side effects will be different among individuals. Therefore, the objective estimation of circadian phases in individuals is required to optimize the timing of medication.

environment requirement for DLMO makes it difficult to perform experimental studies under out-of-laboratory conditions. Third, although DLMO is generally estimated on the basis of a common threshold value of melatonin concentration, melatonin levels not only differ substantially among individuals but also are strongly affected by aging and neurodegenerative disorders. ${ }^{33,34}$ DLMO-based phase estimation therefore has several limitations with respect to interindividual comparisons of circadian-phase differences; however, this method remains a powerful tool for examining intraindividual circadian-phase shifts.

If saliva or blood collection for melatonin measurement is performed every few hours around the clock and under dim light conditions, it is possible to compare circadian phases among individuals whose melatonin levels are in a normal range and similar to one another. However, the sampling frequency needs to be high for circadian-phase estimations using only this marker. In contrast, circadian-phase estimation based on clock gene expression can be performed using several oscillatory markers whose phase intervals are nearly constant, thus enabling highly accurate phase estimation with low sampling frequencies (for example, three times per day).

\section{CIRCADIAN-PHASE ESTIMATION BASED ON PERIPHERAL CLOCK GENE EXPRESSION}

Almost every cell in the body contains a cell-autonomous circadian clock. ${ }^{6}$ Therefore, peripheral clocks can be used to estimate the human circadian phase because they behave synchronously with the central clock in the suprachiasmatic nucleus, ${ }^{35,36}$ although there is a phase difference between the peripheral and central clocks. A method using detached oral mucosa has been reported; however, we have not been able to obtain reliable results by using this method, thus suggesting that this method may require special techniques. ${ }^{37} \mathrm{We}$ found that almost all of the cells in the detached oral mucosa were dead, and the purified RNA was therefore highly fragmented. Later, a method using blood cells taken from a peripheral vein was reported. ${ }^{38}$ Although blood collection using a syringe is invasive, this approach is routine in clinical settings. However, physical stimuli and time delays due to separation procedures for the collection of white blood cells may affect the levels of expression of the clock genes. Careful overall modifications of the experimental procedures may overcome these

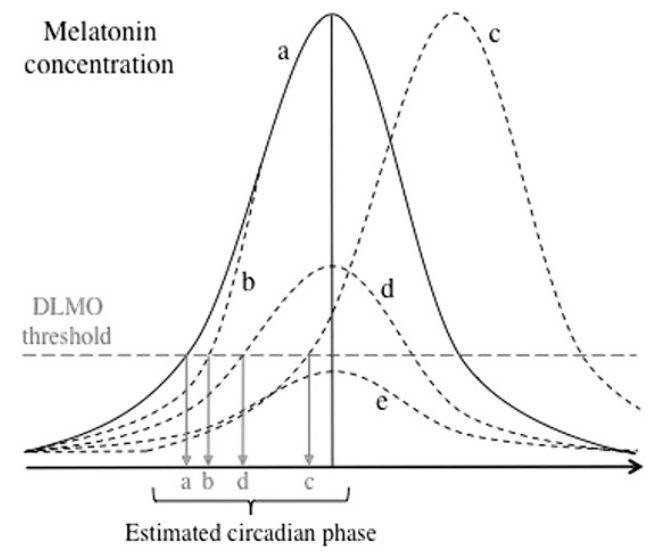

Figure 2 Limitations in dim light melatonin onset (DLMO)-based phase estimation. Curves schematically indicate the diurnal rhythms of melatonin concentrations. A solid curve and dashed curves represent a subject who was appropriate (a, control) and subjects who were inappropriate (b-e) for DLMO-based phase estimations. The red dashed line shows the threshold concentration for the determination of DLMO. The circadian phase in $a, b, d$ and $\mathrm{e}$ is the same, although their calculated DLMOs are not the same. (a) A control example showing that the DLMO method works well with a dim light condition for a subject whose melatonin concentration is sufficient for a reliable determination of DLMO. (b) An example showing that the DLMO method does not work well because the melatonin concentration rapidly decreases in response to a transient light exposure because of a poor dim light condition. (c) An example showing that the duration of specimen collection under a dim light condition must be extended to more than 5-6 h because a rough prediction of DLMO is difficult because of the subject's irregular sleep-wake habits. (d) An example showing that phase estimation does not work well because the melatonin secretion levels differ markedly between individuals. (e) An example showing that the DLMO method does not work at all because the melatonin secretion dramatically decreases because of aging and neurodegenerative disorders. The DLMO-based method has limitations with respect to the interindividual comparison of circadian phases. A full color version of this figure is available at the Hypertension Research journal online.

technical problems; however, we have not been able to obtain reproducible results from either of these methods. As a result, we decided to focus our efforts on a different source of peripheral tissue.

\section{BENEFITS AND LIMITATIONS OF CIRCADIAN-PHASE ESTIMATION BY USING HAIR FOLLICLE CELLS}

For the circadian-phase estimation based on clock gene expression, living cells must be collected with minimal damage to the human subjects. We therefore targeted cells attached to plucked hair roots and attempted to measure clock gene expression in these samples by using the conventional real-time PCR method (Figure 3). ${ }^{39}$ Total RNA purified from the scalp hair follicle cells exhibits two distinguishable absorbance peaks: the $18 \mathrm{~S}$ and $28 \mathrm{~S}$ rRNA signals. One advantage of this method is that not only the quality but also the quantity of RNA is comparatively high (in Japanese male subjects, 200-1000 ng per hair is possible), and commonly used reverse transcriptional methods are applicable. The expression levels of Period3 (Per3), Nr1d1 (Rev-erb $\alpha$ ) and $\operatorname{Nr} 1 d 2($ Rev-erb $\beta)$ genes exhibit clear circadian oscillation with reliable reproducibility. Mathematical phase estimation is performed by calculating a cosine curve with at least three different time points for the expression levels of Per3, Nr1d1 and Nr1d2. Inter- and intraindividual comparisons of circadian phases can be performed on the basis of the peak time of calculated cosine curves.

High-quality total RNA can be extracted from both scalp and facial hair follicles, and reproducible results can be obtained from smaller 


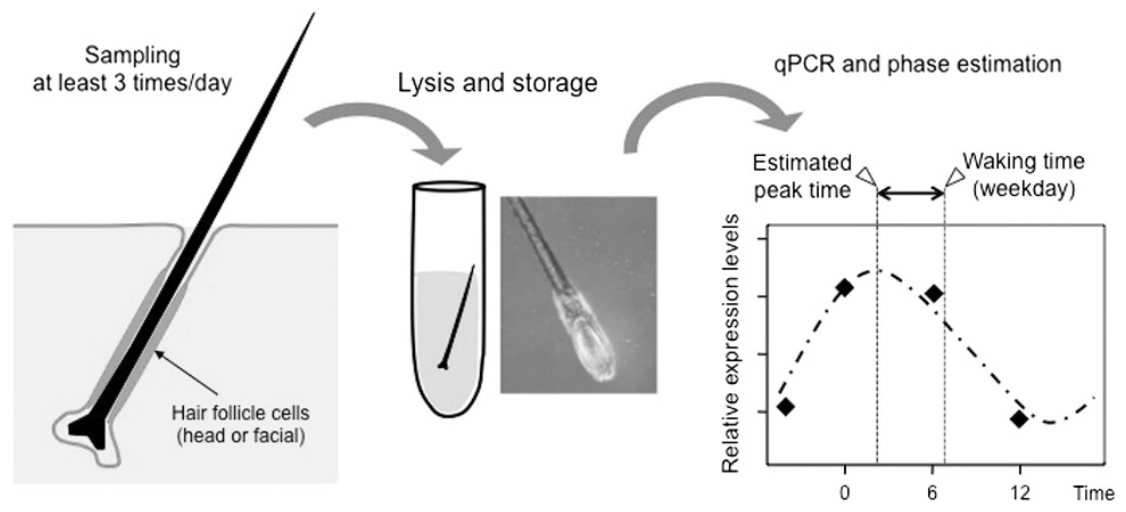

- No special technique, low cost, high reproducibility -

Figure 3 Circadian-phase estimation using hair follicle cells. The human circadian phase can be determined by using the hair follicle cells attached to the scalp or facial hair roots by real-time PCR. The hair should be sampled more than three times per day. At each sampling time point, the quantification of clock gene expression requires more than $200 \mathrm{ng}$ of total RNA. For Japanese subjects, 1-10 strands of hair need to be collected. The Per3, Nr1d1 and $\mathrm{Nr} 1 \mathrm{~d} 2$ genes exhibit circadian fluctuations in transcription with good reproducibility. We previously reported that the intervals among the peak expression times of these three genes were constantly maintained with only small interindividual variation. From the expression levels of Per3, NrId1 and NrId2 at more than three different time points, the peak times are determined by a cosine curve fitted with the least-squares method. Circadian misalignment can be evaluated by making a comparison between the mathematically estimated peak times and the lifestyle time schedules of the subjects (in particular, waking times on weekdays/workdays). A full color version of this figure is available at the Hypertension Research journal online.

numbers of facial hairs than scalp hairs. With recent improvements in this method, for Japanese men, 1-10 scalp hairs or 1-5 facial hairs are required for each time point. For Japanese women, 1-10 scalp hairs are typically sufficient, although more hair may be required in some cases. This uncertainty in sample collection is the primary limitation of this method. Specifically, no information is available on potential race-based differences in the number of hair roots required to obtain a sufficient amount of total RNA for analysis. All specimens must be visually evaluated to confirm whether a sufficient number of hair follicle cells are attached to the roots. The detection efficiency may be further increased not only by the improvement of hair collection and the refinement of the protocol for RNA extraction but also by re-evaluating the reverse-transcription enzyme and the primers/probes for PCR.

As with any peripheral markers, how accurately the circadian phase of the hair follicle cells reflects the circadian phase of the whole body or the suprachiasmatic nucleus is unclear. Under steady-state conditions, such as regular sleep-wake cycles and scheduled meal times, peripheral clocks synchronize well with the central clock. However, desynchronization may transiently occur during phase shifts induced by rescheduling light-dark cycles or food-intake times. ${ }^{40}$ Our previous experiments on the effect of a gradual advance in a daily life schedule (waking time, meal times and bedtime) on circadian gene expression in the hair follicle cells have suggested that the rate of phase shift is relatively low. ${ }^{39}$ Therefore, although a few mouse peripheral tissues have shown rapid circadian phase shifts in response to time-restricted feeding, ${ }^{41}$ resulting in transient desynchronization from the suprachiasmatic nucleus and other peripheral clocks, the same may not be true of hair follicle cells.

\section{MASKED CIRCADIAN RHYTHM SLEEP DISORDER}

The phase estimation method based on clock gene expression may help improve the field of clinical sleep medicine. Sleep disorders attributed to circadian dysfunction are called 'circadian rhythm sleep disorders' and can be subdivided into several types: delayed sleep-phase type, advanced sleep-phase type, non-24-h sleep-wake type and irregular sleep-wake type..$^{24,25,42,43}$ A large proportion of individuals afflicted with circadian rhythm sleep disorders have delayed sleep-phase syndrome. ${ }^{44}$ Because the modern life environment and lifestyle habits have exacerbated circadian maladjustment, the main contributor to delayed sleep-phase syndrome is thought to be minor but chronic jet lag in daily lives. This so-called 'social jet lag', ${ }^{45}$ which has rapidly spread around the world in recent years, probably because of rapid increases in late-night use of light-emitting devices, may be attributed to the prevalence of sleep problems. However, circadian rhythm sleep disorders have been reported to affect $~ 3 \%$ of the population. ${ }^{46-49}$ This unexpectedly low incidence rate may be the result of the masking of circadian misalignment because of the forced adaptation to social time schedules; people must wake up in the morning in time for work or school, despite any circadian sleep problems (Figure 4). Although unsubstantiated, given the modern life environment and lifestyle habits, the number of people potentially suffering from masked circadian rhythm sleep disorders may be substantial, particularly among younger generations.

\section{POSSIBLE APPLICATION OF THE HAIR FOLLICLE METHOD TO CLINICAL SLEEP MEDICINE}

Masked circadian rhythm sleep disorders are difficult to detect in clinical settings by using the currently available tools and methods. For example, sleep diaries and actograms obtained over several weeks are useful for diagnosing typical circadian rhythm sleep disorders, but cannot provide reliable information on masked circadian rhythm disorders. However, the method based on clock gene expression in hair follicle cells may aid in the objective and rapid clinical detection of circadian sleep problems. Specifically, masked circadian misalignment can be evaluated by comparing the internal phase, estimated by using the hair follicle method, with lifestyle time schedules; in particular, the waking time of individuals on weekdays/workdays is usually strictly limited by social factors and is susceptible to misalignment with the internal circadian phase. ${ }^{50}$ Our previous data in healthy subjects have indicated that Per3 mRNA levels peak $\sim 1-4 \mathrm{~h}$ before the subjects' habitual waking times. ${ }^{39}$ The possibility of a masked circadian rhythm sleep disorder therefore increases when the estimated peak times fall outside of this range. 
a

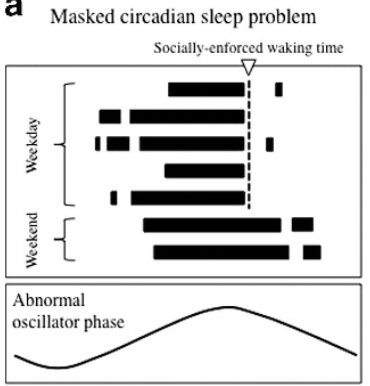

b

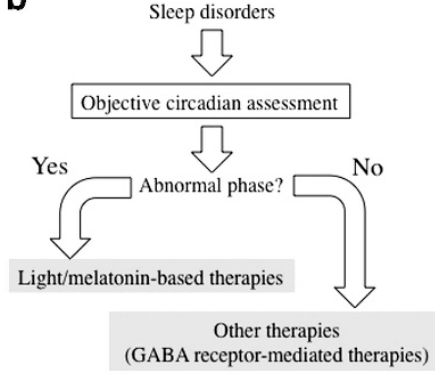

Figure 4 Masked circadian rhythm sleep disorder. (a) It may be difficult to prevent the underdiagnosis of circadian rhythm sleep disorders with the current clinical tools and methods because circadian misalignment can be masked because of forced adaptation to social time schedules. The establishment of methods for the assessment of the human circadian phase may help in the rapid clinical detection of socially masked circadian sleep problems. (b) A schematic representation of the possible contribution of objective circadian phase estimation methods to clinical sleep medicine. Effective phase estimation methods may improve the treatment strategy for sleep problems by increasing the accuracy of the methods used for the diagnosis of circadian rhythm sleep disorders.

In the clinical treatment of masked circadian rhythm sleep disorders, forcing an active-phase brain to fall asleep by administering general sleep drugs, such as GABA receptor agonists, may be counterproductive in some cases. Instead, the circadian phase should be correctly adjusted through bright light exposure and/or administration of melatonin receptor agonists ${ }^{42,51,52}$ to help the internal sleep phase synchronize with the patients' actual bedtime. Importantly, the effect of light and melatonin (melatonin derivatives) is known to be 'circadian phase-responsive', $24,25,29,32,43$ and the time of day of the treatment needs to be objectively determined for each patient on the basis of the internal circadian-phase estimation.

\section{CONCLUSIONS}

Many studies have found that chronic misalignment between circadian and environmental/social rhythms carries a significant risk of various disorders. However, irregular sleep-wake cycles and circadian maladjustment often cause 'social jet lag' in our daily lives. The establishment of objective and convenient circadian-phase estimation methods in clinical settings will greatly contribute not only to resolving this global health problem but also to performing effective chronomedicine. Traditional melatonin-based methods have limitations with respect to circadian-phase evaluation; however, our estimation method based on clock gene expression in hair follicle cells may be able to compensate for these limitations.

\section{CONFLICT OF INTEREST}

The authors declare no conflict of interest.

1 Dibner C, Schibler U, Albrecht U. The mammalian circadian timing system: organization and coordination of central and peripheral clocks. Annu Rev Physiol 2010; 72: 517-549.

2 Panda S, Hogenesch JB, Kay SA. Circadian rhythms from flies to human. Nature 2002; 417: 329-335.

3 Reppert SM, Weaver DR. Coordination of circadian timing in mammals. Nature 2002; 418: 935-941.

4 Partch CL, Green CB, Takahashi JS. Molecular architecture of the mammalian circadian clock. Trends Cell Biol 2014; 24: 90-99.

5 Gamble KL, Berry R, Frank SJ, Young ME. Circadian clock control of endocrine factors. Nat Rev Endocrinol 2014; 10: 466-475.

6 Gachon F, Nagoshi E, Brown SA, Ripperger J, Schibler U. The mammalian circadian timing system: from gene expression to physiology. Chromosoma 2004; 113: 103-112.

7 Doherty CJ, Kay SA. Circadian control of global gene expression patterns. Annu Rev Genet 2010; 44: 419-444.

8 Bass J, Takahashi JS. Circadian integration of metabolism and energetics. Science 2010; 330: 1349-1354.

9 Asarnow LD, Soehner AM, Harvey AG. Circadian rhythms and psychiatric illness. Curr Opin Psychiatry 2013; 26: 566-571.

10 Dibner $\mathrm{C}$, Schibler U. Circadian timing of metabolism in animal models and humans. J Intern Med 2015; 277: 513-527.

11 Sahar S, Sassone-Corsi P. Circadian clock and breast cancer: a molecular link. Cell Cycle 2007; 6: 1329-1331.

12 Masri S, Kinouchi K, Sassone-Corsi P. Circadian clocks, epigenetics, and cancer. Curr Opin Oncol 2015; 27: 50-56.

13 Lowrey PL, Takahashi JS. Mammalian circadian biology: elucidating genome-wide levels of temporal organization. Annu Rev Genomics Hum Genet 2004; 5: 407-441.

14 Miller BH, Takahashi JS. Central circadian control of female reproductive function. Front Endocrinol (Lausanne) 2013; 4: 195.

15 Kovanen L, Donner K, Kaunisto M, Partonen T. CRY1, CRY2 and PRKCDBP genetic variants in metabolic syndrome. Hypertens Res 2015; 38: 186-192.

16 Roenneberg T, Kantermann T, Juda M, Vetter C, Allebrandt KV. Light and the human circadian clock. Handb Exp Pharmacol 2013; 217: 311-331.

17 Bedrosian TA, Fonken LK, Nelson RJ. Endocrine effects of circadian disruption. Annu Rev Physiol 2016; 78: 109-131.

18 Cho Y, Ryu SH, Lee BR, Kim KH, Lee E, Choi J. Effects of artificial light at night on human health: a literature review of observational and experimental studies applied to exposure assessment. Chronobiol Int 2015; 32: 1294-1310.

19 Arendt J. Shift work: coping with the biological clock. Occup Med (Lond) 2010; 60: 10-20.

20 Roth T. Appropriate therapeutic selection for patients with shift work disorder. Sleep Med 2012; 13: 335-341.

21 Haus EL, Smolensky MH. Shift work and cancer risk: potential mechanistic roles of circadian disruption, light at night, and sleep deprivation. Sleep Med Rev 2013; 17: 273-284.

22 Levi F, Okyar A, Dulong S, Innominato PF, Clairambault J. Circadian timing in cancer treatments. Annu Rev Pharmacol Toxicol 2010; 50: 377-421.

23 Levi F, Schibler U. Circadian rhythms: mechanisms and therapeutic implications. Annu Rev Pharmacol Toxicol 2007; 47: 593-628.

24 Sack RL, Auckley D, Auger RR, Carskadon MA, Wright KP Jr, Vitiello MV, Zhdanova IV. Circadian rhythm sleep disorders: part I, basic principles, shift work and jet lag disorders. An American Academy of Sleep Medicine review. Sleep 2007; 30: 1460-1483.

25 Sack RL, Auckley D, Auger RR, Carskadon MA, Wright KP Jr, Vitiello MV, Zhdanova IV. Circadian rhythm sleep disorders: part II, advanced sleep phase disorder, delayed sleep phase disorder, free-running disorder, and irregular sleep-wake rhythm. An American Academy of Sleep Medicine review. Sleep 2007; 30: 1484-1501.

26 Benloucif S, Burgess HJ, Klerman EB, Lewy AJ, Middleton B, Murphy PJ, Parry BL, Revell VL. Measuring melatonin in humans. J Clin Sleep Med 2008; 4: 66-69.

27 Obayashi K, Saeki K, Tone N, Kurumatani N. Relationship between melatonin secretion and nighttime blood pressure in elderly individuals with and without antihypertensive treatment: a cross-sectional study of the HEIJO-KYO cohort. Hypertens Res 2014; 37: 908-913.

28 Lewy AJ. Melatonin and human chronobiology. Cold Spring Harb Symp Quant Biol 2007; 72: 623-636.

29 Keijzer H, Smits MG, Duffy JF, Curfs LM. Why the dim light melatonin onset (DLMO) should be measured before treatment of patients with circadian rhythm sleep disorders. Sleep Med Rev 2014; 18: 333-339.

30 Stehle JH, von Gall C, Korf HW. Organisation of the circadian system in melatonin-proficient $\mathrm{C} 3 \mathrm{H}$ and melatonin-deficient $\mathrm{C} 57 \mathrm{BL}$ mice: a comparative investigation. Cell Tissue Res 2002; 309: 173-182.

31 Stehle JH, von Gall C, Korf HW. Melatonin: a clock-output, a clock-input. J Neuroendocrinol 2003; 15: 383-389.

32 Duffy JF, Czeisler CA. Effect of light on human circadian physiology. Sleep Med Clin 2009; 4: 165-177.

$33 \mathrm{Wu}$ YH, Swaab DF. Disturbance and strategies for reactivation of the circadian rhythm system in aging and Alzheimer's disease. Sleep Med 2007; 8: 623-636.

34 Pandi-Perumal SR, Trakht I, Srinivasan V, Spence DW, Maestroni GJ, Zisapel N, Cardinali DP. Physiological effects of melatonin: role of melatonin receptors and signal transduction pathways. Prog Neurobiol 2008; 85: 335-353.

35 Schibler U, Sassone-Corsi P. A web of circadian pacemakers. Cell 2002; 111 : 919-922.

36 Mohawk JA, Green CB, Takahashi JS. Central and peripheral circadian clocks in mammals. Annu Rev Neurosci 2012; 35: 445-462.

37 Bjarnason GA, Jordan RC, Wood PA, Li Q, Lincoln DW, Sothern RB, Hrushesky WJ, Ben-David Y. Circadian expression of clock genes in human oral mucosa and skin: association with specific cell-cycle phases. Am J Pathol 2001; 158: 1793-1801.

38 Boivin DB, James FO, Wu A, Cho-Park PF, Xiong H, Sun ZS. Circadian clock genes oscillate in human peripheral blood mononuclear cells. Blood 2003; 102: 4143-4145. 
39 Akashi M, Soma H, Yamamoto T, Tsugitomi A, Yamashita S, Yamamoto T, Nishida E, Yasuda A, Liao JK, Node K. Noninvasive method for assessing the human circadian clock using hair follicle cells. Proc Natl Acad Sci USA 2010; 107: 15643-15648.

40 Cermakian N, Sassone-Corsi P. Environmental stimulus perception and control of circadian clocks. Curr Opin Neurobiol 2002; 12: 359-365.

41 Asher G, Sassone-Corsi P. Time for food: the intimate interplay between nutrition, metabolism, and the circadian clock. Cell 2015; 161: 84-92.

42 Pandi-Perumal SR, Trakht I, Spence DW, Srinivasan V, Dagan Y, Cardinali DP. The roles of melatonin and light in the pathophysiology and treatment of circadian rhythm sleep disorders. Nat Clin Pract Neurol 2008; 4: 436-447.

43 Bjorvatn B, Pallesen S. A practical approach to circadian rhythm sleep disorders. Sleep Med Rev 2009; 13: 47-60.

44 Gradisar M, Crowley SJ. Delayed sleep phase disorder in youth. Curr Opin Psychiatry 2013; 26: 580-585.

45 Foster RG, Peirson SN, Wulff K, Winnebeck E, Vetter C, Roenneberg T. Sleep and circadian rhythm disruption in social jetlag and mental illness. Prog Mol Biol Trans/ Sci 2013; 119: 325-346.
46 Sivertsen B, Pallesen S, Stormark KM, Boe T, Lundervold AJ, Hysing M. Delayed sleep phase syndrome in adolescents: prevalence and correlates in a large population based study. BMC Public Health 2013; 13: 1163.

47 Paine SJ, Fink J, Gander PH, Warman GR. Identifying advanced and delayed sleep phase disorders in the general population: a national survey of New Zealand adults. Chronobiol Int 2014; 31: 627-636.

48 Sivertsen B, Harvey AG, Pallesen S, Hysing M. Mental health problems in adolescents with delayed sleep phase: results from a large population-based study in Norway. J Sleep Res 2015; 24: 11-18.

49 Kim MJ, Lee JH, Duffy JF. Circadian rhythm sleep disorders. J Clin Outcomes Manag 2013; 20: 513-528.

50 Wittmann M, Dinich J, Merrow M, Roenneberg T. Social jetlag: misalignment of biological and social time. Chronobiol Int 2006; 23: 497-509.

51 Reid KJ, Zee PC. Circadian rhythm disorders. Semin Neurol 2009; 29: 393-405.

52 van Geijlswijk IM, Korzilius HP, Smits MG. The use of exogenous melatonin in delayed sleep phase disorder: a meta-analysis. Sleep 2010; 33 1605-1614. 